\title{
RESEARCH NOTES \\ ON HYPER-REFLEXIVITY OF SOME OPERATOR SPACES
}

\author{
H. S. MUSTAFAYEV \\ Institute of Mathematics \& Mechanics \\ Azerbaijan \\ Baku, RUSSIA \\ Ondokuz Mayis Universitesi Lojmanlari \\ Kurupelit Kampusu, K-Blok, No 1 \\ 55139 Samsun, TURKEY
}

(Received May 4, 1994 and in revised form March 8, 1995)

\begin{abstract}
In the present note, we define operator spaces with $n$-hyper-reflexive property, and prove $n$-hyper-reflexivity of some operator spaces
\end{abstract}

KEY WORDS AND PHRASES. Operator algebras on Hilbert spaces, reflexivity, hyper-reflexivity 1992 AMS SUBJECT CLASSIFICATION CODES. 47D25

\section{INTRODUCTION}

Let $H$ be a Hilbert space, and $B(H)$ be the algebra of all bounded linear operators on $H$ It is well known that $B(H)$ is the dual space of the Banach space of trace class operators If $T \in B(H)$, $R \subset B(H)$, and $n$ is a positive integer, then $H^{(n)}$ denotes the direct sum of $n$ copies of $H, T^{(n)}$ denotes the direct sum of $n$ copies of $T$ acting on $H^{(n)}$ and $R^{(n)}=\left\{T^{(n)} \mid T \in R\right\} \quad$ Let $P(H)$ be the set of all orthogonal projections in $B(H)$ For any subspace $R \subset B(H)$, we will denote by $l(R)$ the collection of all maximal elements of the set

$$
\{(Q, P) \mid(Q, P) \in P(H) \times P(H), Q R T=0\}
$$

with respect to the natural order It can be seen that if $R$ is a unital subalgebra of $B(H)$, then

$$
l(R)=\{1-P, P) \mid P \in \text { lat } R\}
$$

where lat $R$ is lattice of all invariant subspace of $R$ Recall that an algebra $R \subset B(H)$ is transitive if lat $R\{0,1\}$, and reflexive if the only operators that leave invariant all of the invariant subspaces of $R$ are the operators belonging to $R$ Generalizing this notion, we say that an operator space $R \subset B(H)$ is transitive if $l(R)=\{(0,1),(1,0)\}$ (this is equivalent to $\overline{R x}=H$ for any $x \in H-\{0\}$ ), and is reflexive if

$$
R=\{T \in B(H) \mid Q T P=0 \text { for every }(Q, P) \in l(R)\} .
$$

In other words, $R$ is reflexive if the seminorms $d(T, R)$ and $\sup \{\|Q T P\| \mid(Q, P) \in P(R)\}$ vanish on $R$ simultaneously, where $d(T, R)$ is the distance from $T$ to $R$ It can be seen that

$$
d(T, R) \geq \sup \{\|Q T P\| \mid(Q, P) \in l(R)\}
$$

for any $T \in B(H)$.

Reflexive operator space $R \subset B(H)$ is called hyper-reflexive if there exists some constant $C \geq 1$ such that

$$
d(T, R) \leq C \sup \{\|Q T P\| \mid(Q, P) \in l(R)\}
$$

for any $T \in B(H)$, (see [1-5]).

In [4], an example of non hyper-reflexive operator algebras is constructed 
In the present note, we define operator spaces with $n$-hyper-reflexive property, and prove $n$-hyperreflexivity of some operator spaces

The operator space $R \subset B(H)$ is called $n$-reflexive if $R^{(n)}$ is reflexive lt can be shown that

$$
d(T, R) \geq \sup \left\{\left\|Q T^{(n)} P\right\| \mid(Q, P) \in l\left(R^{(n)}\right)\right\}
$$

for any $T \in B(H)$ and $n \in N$

We say that the $n$-reflexive operator space $R \subset B(H)$ is $n$-hyper-reflexive if there exists some constant $C \geq 1$ such that

$$
d(T, R) \leq C \sup \left\{\left\|Q T^{(n)} P\right\| \mid(Q, P) \in l\left(R^{(n)}\right)\right\}
$$

for any $T \in B(H)$

It is easily seen that if $R$ is $n$-reflexive ( $n$-hyper-reflexive) then it is $k$-reflexive ( $k$-hyper-reflexive) for every $k>n$

\section{MAIN RESULT}

Let us consider in $B(H)$ the following operator equation

$$
\sum_{\imath=1}^{n} A_{\imath} X B_{\imath}=X
$$

The space of all solutions of the equation $(21)$ will be denoted by $R$

PROPOSITION 1. $R$ is $(n+1)$-reflexive

PROOF. For given any $x, y \in H-\{0\}$, put

$$
x=\left(B_{1} x, \ldots, B_{n} x, x\right) \in H^{(n+1)} \quad \text { and } \quad y=\left(A_{1}^{*} y, \ldots, A_{n}^{*} y,-y\right) \in H^{(n+1)} .
$$

Let $P_{x}$ and $Q_{y}$ be the one-dimensional projections on one-dimensional subspaces $\left\{C_{x}\right\}$ and $\left\{C_{y}\right\}$ respectively From (2.1), we have $\left(Q_{y}, P_{x}\right) \in l\left(R^{(n+1)}\right)$ On the other hand, it is easy to see that any $T \in B(H)$ is a solution of equation (2.1) if and only if $Q_{y} T^{(n+1)} P_{x}=0$ This completes the proof.

We will assume that, in case $n>1$, the coefficients of equation (2 1) satisfy the following conditions

$$
\left\|A_{\imath}\right\| \leq 1, \quad\left\|B_{\imath}\right\| \leq 1, A_{\imath} A_{\jmath}=B_{\imath} B_{\jmath}=0(1 \leq i<j \leq n) .
$$

The purpose of this note is to prove the following.

THEOREM 2. The space $R$ of all solutions of (2.1) and (2 2) is $(n+1)$-hyper-reflexive.

To prove Theorem 2 we need some preliminary results.

Let $Y$ be a Banach space with $Y^{*}=X$ and $S$ be a weak ${ }^{*}$ continuous linear operator on $X$ with uniformly bounded degree, $\left\|S^{n}\right\| \leq C(n \in N)$ Denote by $E$ the space of all fixed points of $S$, $E=\{x \in X \mid S x=x\}$ If $x_{0} \in E$, then for any $x \in X$ we have

and consequently

$$
\left\|S^{n} x-x\right\|=\left\|S^{n}\left(x-x_{0}\right)-\left(x-x_{0}\right)\right\| \leq(C+1)\left\|x-x_{0}\right\|
$$

$$
d(x, E) \geq \frac{1}{C+1} \sup _{n}\left\|S^{n} x-x\right\|
$$

PROPOSITION 3. Under the above assumptions,

$$
d(x, E) \leq \sup _{n}\left\|S^{n} x-x\right\|
$$

for any $x \in X$

PROOF. Since $E$ is a weak ${ }^{*}$ closed subspace of $X$, there exists a subspace $M \subset Y$ such that $M^{\perp}=E$, where $M^{\perp}$ is the annihilator of $M$ It can be seen that the set $\{T y-y \mid y \in Y\}$ weak ${ }^{*}$ generates $M$, where $T$ is the preadjoint of $S$, that is, $T^{*}=S$. Let $x \in X$ and let $K(x)$ be the weak closure of the convex hull of the set $\left\{S^{n} x \mid n \in N\right\}$ By Alaoğlu's theorem, $K(x)$ is weak compact We will show that $K(x) \cap E \neq \oslash$ for any $x \in X \quad$ Suppose that $K(x) \cap E=\oslash$. By Hahn-Banach separating theorem, there exists $y_{0} \in M$ such that 


$$
\inf _{a \in K(x)}\left|\left\langle a, y_{o}\right\rangle\right|=\sigma>0
$$

where $\langle$,$\rangle is the duality between X$ and $Y$

Put

$$
x_{n}=\frac{1}{n} \sum_{k-1}^{n} S^{k} x \text {. }
$$

Then $x_{n} \in K(x)$ and $\left\|x_{n}\right\| \leq C\|x\|$ Now, we will prove that

$$
\lim _{n}\left|\left\langle x_{n}, y_{0}\right\rangle\right|=0 \text {. }
$$

Since $\left(x_{n}\right)$ is a bounded set, it is sufficient to prove the equality (2 3$)$ in case $y_{0}=T y-y,(y \in Y)$ In that case

$$
\left\langle x_{n}, T y-y\right\rangle=\left\langle S x_{n}-x_{n}, y\right\rangle=\frac{1}{n}\left\langle S^{n+1} x-S x, y\right\rangle \rightarrow 0 .
$$

Now, suppose that $\left\|S^{n} x-x\right\| \leq \delta$ for some $\delta>0$ and any $n \in N \quad$ It is easy to see that $\|a-x\| \leq \delta$ for any $a \in K(x)$ Let $a_{0} \in K(x) \cap E$ Then $\left\|a_{0}-x\right\| \leq \delta$ and consequently $d(x, E) \leq \delta$

PROOF. OF THEOREM 2. For any $A \in B(H)$ we denote by $L_{A}$ and $R_{A}$ the left and right multiplication operators $L_{A}: X \rightarrow A X, R_{A}: X \rightarrow X A$ on $B(H)$ respectively Then we may write equation (2 1) as

$$
\left(\sum_{\imath=1}^{n} L_{A_{i}} R_{B_{\imath}}\right) X=X
$$

Thus, the solution space $R$ of (2 1) coincide with the set of all fixed points of the operator

$$
S=\sum_{\imath=1}^{n} L_{A_{i}} R_{B_{i}}
$$

It is easily seen that $S$ is a weak ${ }^{*}$ continuous linear operator on $B(H)$ Moreover, under assumption (2 2), it can be shown (by induction) that

$$
S^{k}=\sum_{i=1}^{n} L_{A_{i}^{k}} R_{B_{i}^{k}}
$$

and consequently $\left\|S^{K}\right\| \leq n$

By Proposition 3, for any $T \in B(H)$ we have

$$
\begin{aligned}
d(T, R) & \leq \sup _{k}\left\|S^{k}(T)-T\right\|=\sup _{k}\left\|\sum_{\imath=1}^{n} A_{\imath}^{k} T B_{\imath}^{k}-T\right\| \\
& =\sup _{k} \sup _{\|x\| \leq 1,\|y\| \leq 1}\left|\sum_{\imath=1}^{n}\left(T B_{\imath}^{k} x, A_{\imath}^{* k} y\right)-(T x, y)\right| .
\end{aligned}
$$

For $\|x\| \leq 1$ and $\|y\| \leq 1$, let $x_{k}=\left(B_{1}^{k} x, \ldots, B_{n}^{k} x, x\right), y_{k}=\left(A_{1}^{* k} y, \ldots, A_{n}^{* k} y,-y\right) \quad$ It can be seen that

$$
\left(R^{(n+1)} x_{k}, y_{k}\right)=0 \text { and }\left\|x_{k}\right\|^{2} \leq n+1,\left\|y_{k}\right\|^{2} \leq n+1(k \in N) \text {. }
$$

Therefore

$$
d(T, R) \leq(n+1) \sup \left\{\left|\left(T^{(n+1)} x, y\right)\right| \mid\left(R^{(n+1)} x, y\right)=0,\|x\|=\|y\|=1\right\} .
$$

Let $P_{x}, Q_{y}$ be the one-dimensional projections (as in the proof of Proposition 1) Then we obtain 


$$
\begin{aligned}
d(T, R) & \leq(n+1) \sup \left\{\left\|Q_{y} T^{(n+1)} P_{x}\right\| \mid Q_{y} R^{(n+1)} P_{x}=0\right\} \\
& \leq(n+1) \sup \left\{\left\|Q T^{(n+1)} P\right\| \mid(Q, P) \in l\left(R^{(n+1)}\right)\right\} .
\end{aligned}
$$

This completes the proof

COROLLARY 4. Let $A, B \in B(H)$ with $\|A\| \leq 1,\|B\| \leq 1$ Then, the solution space $R$ of the equation

$$
A X B=X
$$

is 2-hyper-reflexive with constant $C=2$

Generally speaking, the solution space of equation (2 4) may be reflexive For example, if $\mathrm{Q}, P \in P(H)$, then the solution space of equation

$$
Q X P=X
$$

is reflexive Hyper-reflexivity (with constant $C=1$ ) of the solution space of equation (2 5) was proved in [3]

Note that the space of all Toeplitz operators $\tau$ coincide with the solution space of $(24)$ in case $A=U^{*}$ and $B=U$, where $U$ is a unilateral shift operator on Hardy space $H^{2}$ [6]

Consequently, $\tau$ is a 2-reflexive by Proposition 1 Using Theorem 2, we can deduce even more

COROLLARY 5. The space of all Toeplitz operators $\tau$ is 2-hyper-reflexive, with constant $C=2$ In other words

$$
d(T, \tau) \leq 2 \sup \left\{\left\|Q T^{(2)} P\right\| \mid(Q, P) \in l\left(\tau^{(2)}\right)\right\}
$$

for any $T \in B\left(H^{2}\right)$

On the other hand we have the following

PROPOSITION 6. The space of all Toeplitz operators $\tau$ is transitive (consequently $\tau$ is not reflexive)

PROOF. Suppose that $\tau$ is nontransitive Then there exists $f, g \in H^{2}-\{0\}$ such that $(T f, g)=0$ for every $T \in \tau$ If we put in last equality $T=U^{n}$ and $T=U^{* n}(n=0,1,2, \ldots)$, then we obtain that the Fourier coefficients of the function $f \bar{g}$ are zero Since $f \bar{g}=0 \mathrm{a} \mathrm{e}$, one of these functions vanishes a e on some subset of the unit sicle with positive Lebesque measure By $F$ and $M$ Riesz uniqueness theorem [6], one of these functions is zero

Hyper-reflexivity of algebras of analytic Toeplit $z$ operators was proved in [5]

\section{REFERENCES}

[1] ARVESON, W., Interpolation problems in algebras, J. Functional Analysis 20 (1975), 208-233.

[2] SHULMAN, V S, Vektor functionals, Spectral Theory of Operators 5 (1984), 192-225 (Russian)

[3] PARROT, S, On a quotient norm and Sz Nagy-Foias lifting theorem, J. Funct. Anal. 30 (1978), 311-328

[4] DAVIDSON, K R and POVER, S C., Failure of the distance formula, J. London Math. Soc. 32 (1985), 157-165

[5] DAVIDSON, K R, The distance to the analytic Toeplitz operators, Illinols J. Math. 31 (1987), 265273

[6] DOUGLAS, R G, Banach Algebra Techniques in Operator Theory, New York, Academic Press (1972)

[7] MUSTAFAYEV, H S and SHULMAN, V.S, On the denseness of vector functionals, Soviet Math. Dokl. 31 (1985), 167-170 (English translation) 


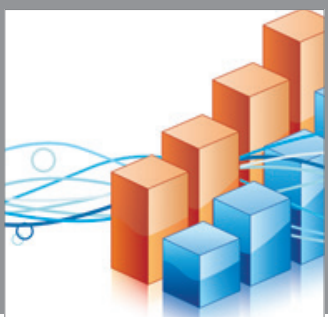

Advances in

Operations Research

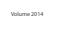

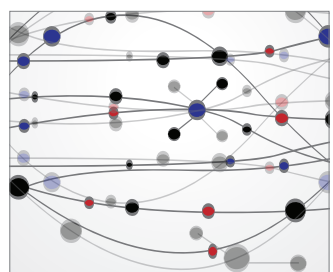

\section{The Scientific} World Journal
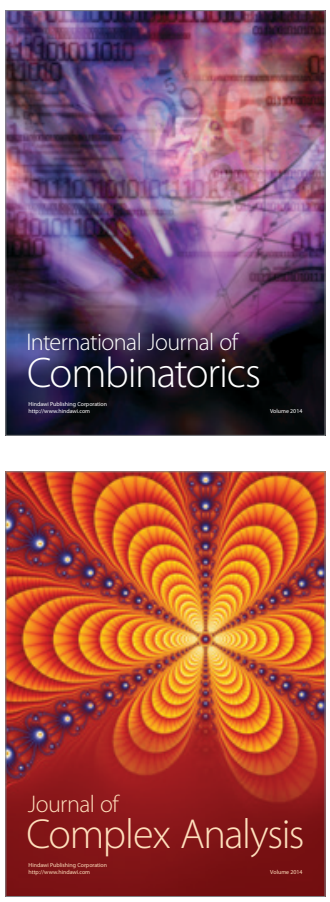

International Journal of

Mathematics and

Mathematical

Sciences
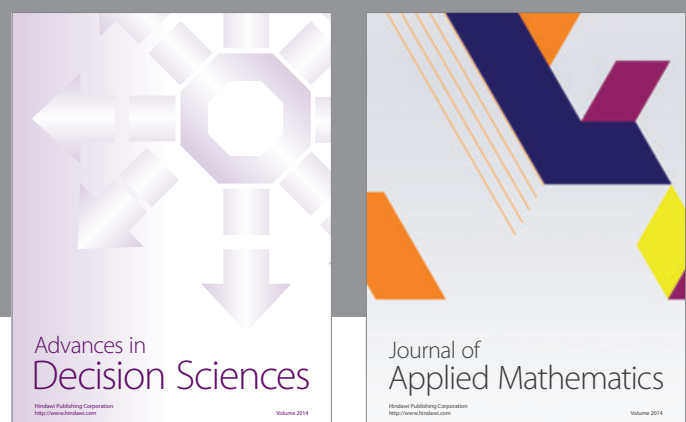

Journal of

Applied Mathematics
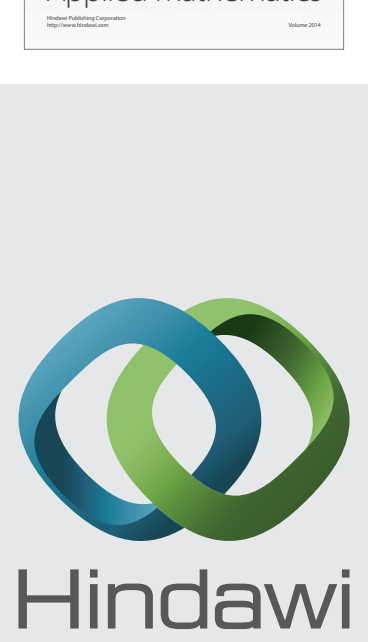

Submit your manuscripts at http://www.hindawi.com
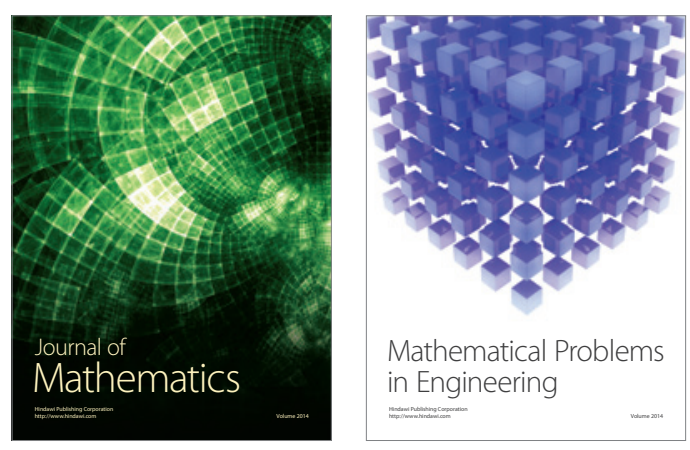

Mathematical Problems in Engineering
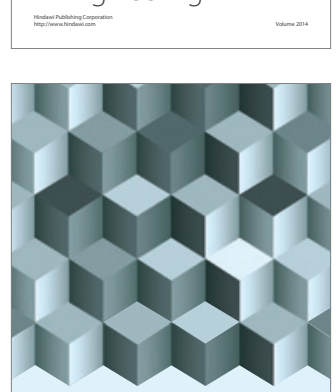

Journal of

Function Spaces
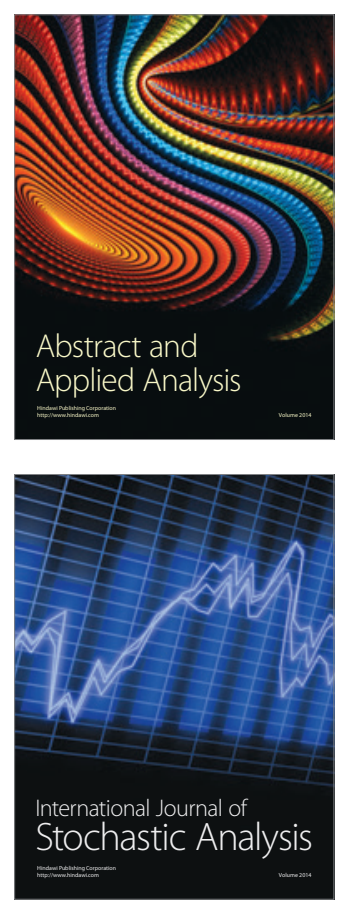

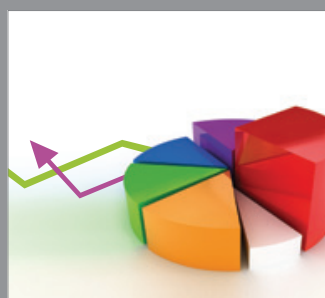

ournal of

Probability and Statistics

Promensencen
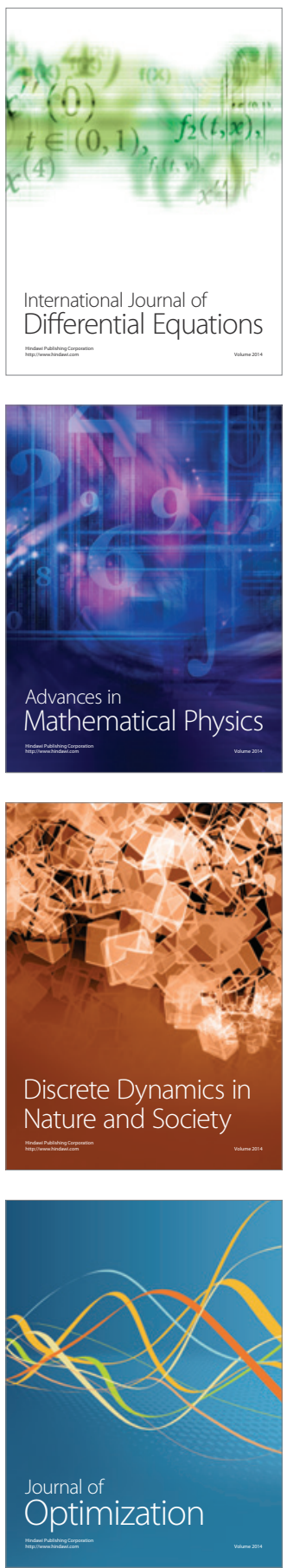